\title{
Endocardite acuta da Staphylococcus haemolyticus meticillino sensibile, con recidiva dopo trattamento da ceppo meticillino resistente
}

\author{
Giacomo Magnani, Faissal Choroma
}

Unità Operativa di Malattie Infettive, Azienda Ospedaliera SMN di Reggio Emilia

\section{RIASSUNTO}

Le endocarditi su valvole native (NVE) da Stafilococchi coagulasi negativi (CoNS) presentano un andamento aggressivo simile a quello da Staphylococcus aureus e sono difficili da trattare per l'alta incidenza da parte dei CoNS di resistenza a oxacillina.Viene riportato un caso di NVE da Staphylococcus haemolyticus meticillino sensibile, con risposta iniziale favorevole al trattamento con oxacillina, ma con successiva recidiva a distanza di tre mesi legata comparsa di ceppo oxacillino-resistente. L'ipotesi è che l'infezione sia stata sostenuta da una popolazione batterica con fenotipo eteroresistente, che in seguito al trattamento antibiotico ha sviluppato resistenza omogenea a oxacillina. Nell'impostare un trattamento per infezioni gravi da Stafilococchi sensibili in vitro ad oxacillina è necessario considerare sempre la presenza di un eteroresistenza.

\section{INTRODUZIONE}

Gli stafilococchi coagulasi negativi (CoNS) sono considerati tra i principali responsabili di sepsi sia comunitarie che nososocomiali (4, 10). Interessano prevalentemente soggetti con deficit immunitari o portatori di devices, pazienti pediatrici o pazienti anziani residenti in strutture di lungo degenza. In uno studio condotto in Lombardia nel 2000 la prevalenza di CoNS tra le infezioni nososomiali è risultata del $7.5 \%(7)$ ed in un studio condotto in 41 ospedali del Nord Italia l'incidenza di sepsi ad essi ascrivibile è risultata del 5.1\% (11). L'incidenza di infezioni da CoNS è peraltro significativamente maggiore in alcune categorie di pazienti, quali ad esempio gli emodializzati (13).

Una delle principali difficoltà nel trattamento delle infezioni sostenute da CoNS è costituita dalla resistenza a meticillina, costantemente presente in oltre il $70 \%$ dei ceppi isolati nelle varie parti del mondo (4). I ceppi nosocomiali associano anche la resistenza verso i fluorochinoloni e gli aminoglicosidi, con necessità di ricorso ai glicopeptidi o a nuovi antibiotici, quali il linezolid. Inoltre è frequente per gli stafilococchi il fenomeno delle eteroresistenza, caratterizzata dalla presenza all'interno di una popolazione sensibile a meticillina, di una popolazione di piccole dimensioni $(0.1-0.01 \%)$ con resistenza di grado elevato a meticillina, che in seguito a trattamento andrà a sostituirsi alla prima facendo fallire il trattamento con $\beta$-lattamici.

L'incidenza dei CoNS, come agenti di endocarditi infettive è andata aumentando negli ultimi decenni e per le endocardite infettive su valvole native (NVE) si colloca intorno al 6\% $(3,9)$. L'evoluzione clinica delle NVE da CoNS è simile a quella da Staphylococcus aureus per quanto concerne la comparsa sia di insufficienza cardia- ca che di ascesso perivalvolare, la necessità di ricorso al chirurgo e l'incidenza di mortalità $(19 \%$ vs $25 \%$ ); molto meno favorevole di quella da Streptococchi viridanti (3).

Qui di seguito, riportiamo un caso di NVE da Staphylococcus haemolyticus meticillino sensibile, con recidiva, dopo oltre tre mesi dalla fine di un trattamento condotto con successo, sostenuta dallo stesso microrganismo ma con fenotipo meticillino-resistente.

\section{CASO CLINICO}

Maschio, di anni 62, con valvulopatia aortomitralica, diagnosticata dall'età di 40 anni, nel luglio 2002 veniva ricoverato per anemia sideropenica e febbre in ambiente medico. Gli veniva posta diagnosi mediante endoscopia digestiva di kissing ulcers bulbari e veniva isolato da $2 / 2$ set di emocolture uno Staphylococcus haemolyticus meticillino sensibilie. Il paziente veniva trattato per 7 giorni con ceftriaxone e dimesso guarito. Dopo circa una settimana di benessere, veniva nuovamente ricoverato, presso il nostro Centro, per ricomparsa di febbre (con puntate iperpirettiche fino a $39^{\circ} \mathrm{C}$ ) e diarrea. All'esame obbiettivo si rilevava soffio olosistolico di $3 / 6$ di intensità sul focolaio aortico. Gli esami di laboratorio evidenziavano VES $1 \mathrm{~h}=88 \mathrm{~mm}, \mathrm{pCR}=5.6 \mathrm{mg} / \mathrm{dl}, \mathrm{Hb}=$ $11.4 \mathrm{~g} / \mathrm{dl}, \mathrm{PLT}=332.000 / \mathrm{mmc}, \mathrm{GB}=5.650 / \mathrm{mmc}$, Neutrofili $=76 \%$; indici di funzionalità epatica, renale, emocoagulativa nei limit di norma. Da 3 set di emocolture veniva isolato uno Staphylococcus haemolyticus meticillino sensibile a oxacillina (MIC $\leq 0.25 \mathrm{mg} / \mathrm{ml}$ ), tetracicline, cotrimossazolo e rifampicina e resistente a fluorochinoloni e aminoglicosidi (Vitek 1; bio-Merieux, Parigi). Negativi risultava la determinazione degli autoanticorpi non organo specifici e la sierologia per Mycoplasma, Legionella, Coxiella e 


\section{Chlamydia.}

L'ecocardiografia transesofagea (TTE) evidenziava una valvola mitralica fibrocalcifica con insufficienza mitralica lieve ed una valvola aortica dismorfica e calcifica con insufficienza moderata; escludeva la presenza di vegetazioni endocardiche. Il paziente veniva trattato con oxacillina 12 gr /die ev in infusione continua, per 6 settimane, con scomparsa della febbre in 3 giorni e normalizzazione degli indici di flogosi $(\mathrm{pCR}=0.60,0.40$ $\mathrm{mg} / \mathrm{dl}$, dopo 30-60 gg).

Dopo oltre 3 mesi di benessere, il 28 novembre 2002, il paziente veniva nuovamente ricoverato per ricomparsa di febbre, artromialgie ed astenia. L'esame obbiettivo cardiaco era invariato; non si rilevavano segni di scompenso emodinamico. Gli esami ematochimici evidenziavano VES $1 \mathrm{~h}=76$, $\mathrm{pCR}=3.25, \mathrm{~GB}=11310 \mathrm{~N}=85 \%$. La TEE: non evidenziava vegetazioni endocardiche; invariata rimaneva l'insufficienza mitralica, mentre era lievemente peggiorata l'insufficienza aortica. Da 3 set di emocolture veniva isolato un ceppo di Staphylococcus haemolyticus resistente a oxacillina $(\mathrm{MIC}=0.5 \mathrm{mcg} / \mathrm{ml})$ gentamicina e fluorochinoloni, sensibile a rifampicina $(\mathrm{MIC}<1 \mathrm{mcg} / \mathrm{ml}) \mathrm{e}$ vancomicina ( $\mathrm{MIC}=2 \mathrm{mcg} / \mathrm{ml}$ ). Si iniziava terapia con vancomicina $1 \mathrm{gr} \times 2 \mathrm{ev}+$ rifampicina 900 mg x 2 per os, che determinava la comparsa della febbre in $3 \mathrm{gg}$ e la normalizzazione dei valori degli indici di flogosi $(\mathrm{pCR}=0.95 \mathrm{md} / \mathrm{dl})$ in 15 giorni. In 24 giornata di terapia, per comparsa di flebite in sede di infusione e difficoltà al reperimento di accessi venosi, vancomicina+rifampicina venivano sostituite con linezolid $600 \mathrm{mg}$ x 2 per os. Il paziente veniva trattato con terapia antibiotica complessivamente per 6 settimane.

In considerazione del peggioramento della insufficienza valvolare aortica e dell'isolamento di un microrganismo multiresistente veniva concordata l'opportunità di intervento di sostituzione valvolare aortica, che veniva effettuato nell'aprile 2003. L'esame istopatologico della valvola asportata confermava la presenza dell'endocardite. Non veniva effettuato esame colturale su tessuto.

\section{DISCUSSIONE E CONCLUSIONI}

Dal caso descritto emergono i seguenti quesiti:

1- quale significato clinico dare all'isolamento di CoNS dalle emocolture?

2- come interpretare i test di sensibilità in vitro e lo shift fenotipico di Staphylococcus haemolyticus per quanto riguarda la sensibilità a oxacillina?

3- quando è opportuno ricorrere a farmaci nuovi, quali il linezolid?

Per quanto attiene al primo quesito, va sottolineato che la maggior parte dei CoNS isolati dalle emocolture sono contaminanti cutanei; ciò espone al rischio di un sovrautilizzo di antibiotici, in particolare di vancomicina, e può rendere difficile decidere se rimuovere o meno un device.

Il numero di flaconi positivi è un buon parametro predittivo di infezione vera, ma va sempre correlato con il dato clinico $(8,14)$. Vengono considerati indicativi di sepsi vera anche la crescita in coltura in meno di 36 ore, la multiresistenza (a più di 6 classi di antibiotici) e l'isolamento di $S$. hominis o di S. haemolyticus (12). Nel nostro caso oltre all'isolamento di Staphylococcus haemolyticus in $3 / 3$ set di emocolture vi erano dati clinici che deponevano per una probabile endocardite, patologia sempre più correlata ad infezione da stafilococchi.

Un caratteristica dei CoNS è la resistenza a meticillina, dovuta alla incorporazione del gene mec-A che codifica per una penicillin-binding-protein, PBP-2a o PBP-2', con bassa affinità per i $\beta$-lattamici. La resistenza a meticillina/oxacillina comporta invariabilmente resistenza anche agli altri $\beta$ lattamici ed a clindamicina, macrolidi, tetracicline, cotrimossazolo aminoglicosidi e fluorochinoloni. Se la presenza del gene $m e c A$ è indispensabile per lo sviluppo di meticillino/oxacillino resistenza, l'espressione fenotipica della resistenza a oxacillina nelle cellule che contengono gene mecA può essere variabile (10). In particolare, va tenuta in considerazione la possibilità di trovarsi di fronte ad un fenotipo eteroresistente, caratterizzato dalla presenza di una popolazione per la maggioranza sensibile a basse concentrazioni a oxacilina, che maschera un piccolo numero di cellule (generalmente $0.1-0.01 \%$ ) resistenti ad una concentrazione di oxacillina $>10 \mu \mathrm{g}$ per ml. Tale eteroresistenza può giustificare l'iniziale successo di una terapia con $\beta$-lattamici e la possibilità di insuccesso in corso di terapia o di recidiva a distanza per la selezione operata dal trattamento di ceppi resistenti a oxacillina, come nel caso da noi riportato. Nell'impostare un trattamento per infezioni gravi stafilococciche con sensibilità in vitro ad oxacillina è, quindi, necessario considerare sempre la presenza di un eteroresistenza ed applicare tutte le metodiche suppletive necessarie per svelare l'eventuale presenza di eteroresistenza.

L'esperienza riportata in letteratura di utilizzo del linezolid nel trattamento delle endocarditi è limitata a 30 casi, con un buon successo sia clinico che microbiologico (1). Secondo quanto affermato anche nelle recenti Linee Guida della British Society for Antimicrobial Chemotherapy il linezolid andrebbe utilizzato come terapia di salvataggio delle endocarditi da microrganismi sensibili, in pazienti intolleranti alla terapia convenzionale o con infezione da ceppi con particolare resistenza (5). 


\section{BIBLIOGRAFIA}

1. Birmingham MC, et al. Linezolid for treatment of multidrugs resistant, Gram positive infections: experience from a compasionate-use program. CID 2003; 36: $159-68$.

2. Chambers HF. Methicillin resistance in staphylococci: molecular and biochemical basis and clinical implications. Clin Microbiol Rev. 1997 Oct; 10(4): 781-91.

3. Chu $\mathrm{VH}$, et al. Native valve endocarditis due to Coagulase-Negative Staphylococci: report of 99 episodes from International Collaboration on Endocarditis merged Database. Clin Infect Dis 2004; 39: 1527-3.

4. Diekema DJ, Pfaller MA, et al. Survey of Infections Due to Staphylococcus Species: Frequency of Occurrence and Antimicrobial Susceptibility of Isolates Collected in the United States, Canada, Latin America, Europe, and the Western Pacific Region for the SENTRY Antimicrobial Surveillance Program, 1997-1999. CID 2001; 32 (Suppl 2): S114-32.

5. Elliot TSJ, et al. Guidelines for antibiotic treatment of endocarditis in aldults:report of the Working Party of the British Society for Antimicrobial Chemotherapy 2004; 54: 971-81.

6. Finan JE, Rosato AE, Dickinson TM, Ko D, Archer GL Conversion of oxacillin-resistant staphylococci from heterotypic to homotypic resistance expression. Antimicrob Agents Chemother. 2002; 46(1): 24-30.

7. Lizioli A, Privitera G, Alliata E, et al. Prevalence of nosocomial infections in Italy: result from the Lombardy survey in $20001 \mathrm{~J}$ Hosp Infect 2003; 54: 141-8.

8. Mirrett S, et al. Relevance of the Number of Positive Bottles in Determining Clinical Significance of Coagulase-Negative Staphylococci in Blood Cultures. J Clin Microbiol 2001 39: 3279-81.

9. Moreillon P, Que Yok-Ai. Infettive endocarditis. Lancet 2004; 363: 139-49.

10. National Nosocomial Infections Surveillance (NNIS) System report, data summary from January 1990-May 1999. Am J Infect Control 1999; 27 (6): 520-32.

11. Panceri et al. Aetiology and Prognosis of Bacteraemia in Italy Epidemiol Infect 2004; 132: 647-54.

12. Ruhe J, Menon A, Mushatt D, Dejace P. Non-epidermidis coagulase-negative staphylococcal bacteremia: clinical predictors of true bacteremia. Eur J Clin Microbiol Infect Dis 2004; 23: 495-8.

13. Scudeller et al, Access Site Related Infections in italian Dialysis: Isolates. ECCMID 2004.

14. Tokars JI. Predictive Value of Blood Cultures Positive for Coagulase-Negative Staphylococci: Implications for Patient Care and Health Care Quality Assurance. CID 2004; 39: 333-41.

Giacomo Magnani

Unità Operativa Malattie Infettive

A.O. Santa Maria Nuova di Reggio Emilia

Via Risorgimento 80 - 42100 Reggio Emilia E-mail: giacomo.magnani@asmn.re.it 